\title{
Multiple access acoustic communication in underwater mobile networks
}

\author{
Christophe Bernard \\ L@bISEN - Yncréa Ouest \\ ISEN Brest \\ Brest, France \\ christophe.bernard@isen-ouest.yncrea.fr \\ Philippe Forjonel \\ L@bISEN - Yncréa Ouest \\ ISEN Brest \\ Brest, France \\ philippe.forjonel@isen-ouest.yncrea.fr
}

\author{
Pierre-Jean Bouvet \\ L@bISEN - Yncréa Ouest \\ ISEN Brest \\ Brest, France \\ pierre-jean.bouvet@isen-ouest.yncrea.fr
}

\author{
Antony Pottier \\ L@bISEN - Yncréa Ouest \\ ISEN Brest \\ Brest, France \\ antony.pottier@isen-ouest.yncrea.fr
}

\begin{abstract}
This article presents a new multiple access technique for underwater acoustic communication in the framework of autonomous underwater vehicle fleet. The multiuser scheme is based on a set of orthogonal waveforms built by combining 2 types of HFM signals allowing at the reception side very simple matched filter based decoding. The proposed multiuser technique provides good resilience against multiuser interference while keeping robustness against underwater acoustic channel impairments like Doppler shift. The implementation of this protocol for the underwater acoustic scenario is described, and performance are analysed and compared against conventional TDMA and CDMA techniques over replayed experimental channels.
\end{abstract}

Index Terms-Underwater acoustic communication; code division multiple access (CDMA); time division multiple access (TDMA).

\section{INTRODUCTION}

Research to understand the underwater environment and to exploit the rich underwater resources has motivated the use of Autonomous Underwater Vehicle (AUV)s which cooperate within a fleet [1]. To control an AUV fleet, Underwater Acoustic (UWA) communications are privileged but channel characteristics are far away from radio frequency channel. In fact, the propagation speed of an underwater acoustic signal is much slower than that of radio signal $\left(3.10^{8} \mathrm{~m} / \mathrm{s}\right.$ for a radio wave against $1500 \mathrm{~m} / \mathrm{s}$ for an acoustic wave) such as delay times of multipath are very long mainly in shallow water areas leading to extensive intersymbol interference, and narrow band frequency selective fading [2]. Moreover, because of this low speed, the transmitted acoustic signal is more vulnerable to the Doppler effect compared to other communication systems. Consequently, even a slow motion between the transmitter/receiver and/or the inherent current wave's motion can bring significant Doppler effect to the transmitted signal [3].

The research work described in this paper was partly funded by the Brittany Region (France) and by Thales DMS France in the framework of the WAVES laboratory.
Traditional methods for multiuser communication in an underwater acoustic channel are Time-Division Multiple Access (TDMA) or Code-Division Multiple Access (CDMA) [4]. The Frequency Divsion Multiple Access (FDMA) protocol can also be used for a multiuser communication but it imposes large guard bands at the spectral level and on the other hand because the signal frequency band already greatly limited by physics is wasted if it is allocated in permanence to a user who does not transmit continuously [2]. TDMA allows several users to share the same frequency channel by dividing the signal into different time slots. Each user uses alternatively its own time slot to transmit data without interfering with other users. However, as the number of users increases, the waiting time per user increases and the user data rate decreases. In CDMA protocol, the different users transmit information data simultaneously through a different spreading sequence for each user. The disadvantage of this method lies in the multiuser interference provided by the nonorthogonality of spreading sequences especially when the user communication channel is selective in time or in frequency. Moreover such effect is increased when the interfered signal power is much larger than received desired signal power, such phenomenon is well known in mobile communication networks as the near-far problem. Another method to mitigate the interference is to use a multiuser detection [5] or MultiUser Multiple-Input Multiple-Output (MU-MIMO) technique combined with Passive Phase Conjugation (PPC) technique [6] but at the prize of higher decoding complexity and users number limitation. Recently, the authors of [7] propose an alternative of CDMA and TDMA by using chirp waveform for UWA multiuser communication. To reduce the multiuser interference, the Virtual Time Reversal Mirror (VTRM) technique is used with a Fractional Fourier Transform (FrFT) at the reception. However, this method requires an estimate of the different channels and is limited to 4 users because of multiuser interference. Recently we have introduced the so-called MultiUser Chirp Spread Spectrum (MU-CSS) 
technique [8] designed to attenuate multiuser interference and to be robust to the effects of the channel by combining Hadamard code with an Hyperbolically Frequency Modulated (HFM) signal thanks to the Gram-Schmidt process [9].

In this article, we propose a multiuser scheme based on a set of orthogonal waveform obtained by combining an HFM signal [10] with a second HFM signal that is narrowband and the resulting signals are made mutually orthogonal owing to the Gram-Schmidt process. Compared to [11], Hadamard codes are replaced by an HFM signal that allows higher robustness to channel effects such as the Doppler spread or multipaths and is more resistant to the Doppler shift. For the experimental results, we will consider a realistic communication by assuming an uplink scenario where a fleet of $N_{u}$ AUVs in motion needs to transmit data to a receiver situated at the sea surface.

The rest of the article is organized as follows: the new multiuser protocol is detailed in section II. In section III, we provide realistic performance results by using the underWater AcousTic channEl Replay benchMARK (Watermark) [12] fed by experiments conducted in the roadstead of Brest, France. Finally, conclusions are drawn in section IV.

In the following, $\langle$.$\rangle the scalar product, (.)^{*}$ the complex conjugate and $u * v$ denotes the convolution product of $u$ by $v$.

\section{SYSTEM MODEL}

\section{A. Transceiver}

Let $g_{i}(t)$ the transmit waveform associated to user $i$ and $T_{s}$ the symbol duration, the baseband transmit signal for user $i$ can be written as:

$$
s_{i}(t)=\sum_{k=1}^{N_{s}} d_{i, k} g_{i}\left(t-k T_{s}\right)
$$

where $d_{i, k}$ are Differential Phase Shift Keying (DPSK) symbols carrying the useful information with $k \in\left[1, N_{s}\right]$. The choice of DPSK is motivated by the rapid fluctuation of UWA channel and thus we can avoid the use of channel equalizers at the receiver side, which are sensitive to outdated channel estimations [13].

If the Doppler shift is known at the receiver, Doppler effect is usually removed to prior decoding by resampling the received baseband signal and compensating phase rotation as follows [14]:

$$
z_{i}(t)=r\left(\frac{t}{1-a_{i}}\right) e^{-j 2 \pi f_{c}\left(\frac{a_{i}}{1-a_{i}}\right) t}
$$

where $a_{i}=\frac{v_{i}}{c}$ is the Doppler shift for the $i$-th user with $v_{i}$ the speed of the $i$-th user and $c \simeq 1500 \mathrm{~m} / \mathrm{s}$ the speed of sound in water.

By assuming perfect time synchronization, information data of the $i$-th user can be estimated by matched filtering $z_{i}(t)$ with transmit waveform of user $i$, followed by integration over a symbol duration (see [8] for more details):

$$
\hat{d}_{i, k}=\int_{-\frac{T_{s}}{2}}^{\frac{T_{s}}{2}} g_{i}^{*}(t) z_{i}\left(t+k T_{s}\right) d t
$$

\section{B. Proposed method}

Unlike [8], in this new method we will replace the Hadamard codes by a family of mutually orthogonal signals that are similar to HFM waveform. In the following, this family will be noted by $\left\{c_{i}(t)\right\}_{i=1}^{N_{u}}$. The orthogonality between $c_{i}(t)$ and $c_{n}(t)$ with $i \neq n$ is obtained by the Simpson's method [15]. For the $i$-th user with $i \in\left[1, N_{u}\right]$ we define:

$$
c_{i}(t)=\frac{1}{\sqrt{T_{s}}} e^{-j 2 \pi k \log \left(1-\frac{t}{i t_{0}}\right) \zeta i^{2}}
$$

where $t_{0}=\frac{T_{s}\left(f_{h}+f_{l}\right)}{2\left(f_{h}-f_{l}\right)}, k=\frac{T_{s} f_{l} f_{h}}{f_{h}-f_{l}}$ is the signal slope, $f_{l}$ is starting frequency, $f_{h}$ is end frequency with $f_{l} \leq f_{h}$ and $T_{s}$ is the duration of the waveform. The Simpson's orthogonality relation is defined for $i \neq n$ by:

$$
\begin{aligned}
\left\langle c_{i}, c_{n}\right\rangle \simeq 0 & \Leftrightarrow e^{-j 2 \pi k \zeta\left(\log \left(1-\frac{-T_{s}}{2 i t_{0}}\right) i^{2}+\log \left(1-\frac{\frac{-T_{s}}{2}}{n t_{0}}\right) n^{2}\right)} \ldots \\
& +4 e^{-j 2 \pi k \zeta\left(\log \left(1-\frac{T_{s}}{2 i t_{0}}\right) i^{2}+\log \left(1-\frac{T_{s}}{2 n t_{0}}\right) n^{2}\right)} \ldots \\
& +e^{-j 2 \pi k \zeta\left(\log \left(1-\frac{\frac{T_{s}}{2}}{i t_{0}}\right) i^{2}+\log \left(1-\frac{T_{s}}{2}\right) n^{2}\right)} \simeq 0
\end{aligned}
$$

where $\zeta \in \mathbb{R}$ is a constant used to determine the orthogonality between the different signals. This constant influences the bandwidth of the different $c_{i}(t)$. In the following we choose a bandwidth $B=4 \mathrm{Khz}$ and therefore we will take:

$$
\zeta= \begin{cases}0.0214 & \text { if } i \text { is even } \\ -0.0214 & \text { otherwise }\end{cases}
$$

We combine the different $c_{i}(t)$ with an HFM signal over the entire bandwidth and to keep the orthogonality between the $c_{i}(t)$ we use the Gram-Schmidt process [9] given for $i>0$ by:

$$
g_{i}(t)=c_{i}(t)+\alpha_{i} e_{i-1}(t)
$$

where:

$$
\alpha_{i}=-\frac{\left\langle c_{i}(t), e_{i-1}(t)\right\rangle}{\left\|e_{i-1}(t)\right\|_{2}^{2}}=-\frac{\int_{\frac{-T_{s}}{2}}^{\frac{T_{s}}{2}} c_{i}(t) e_{i-1}^{*}(t) d t}{\left\|e_{i-1}(t)\right\|_{2}^{2}}
$$

with $c_{i}(t)$ given by the relation (4) and $e_{0}(t)=x(t)$ (this signal $x(t)$ will be excluded from the set later) where $x(t)$ is a HFM signal given by:

$x(t)= \begin{cases}\cos \left(-2 \pi\left(k \log \left(1-\frac{t}{t_{0}}\right)+\frac{f_{l}+f_{h}}{2}\right)\right) & \text { if } \frac{-T_{s}}{2} \leq t \leq \frac{T_{s}}{2} \\ 0 & \text { otherwise }\end{cases}$

In the following, these new waveforms will be called MultiUser Hyperbolically Frequency Modulated (MU-HFM). 


\section{EXPERIMENTAL RESULTS}

\section{A. Channels sounding}

The channel transmission experiment took place in the summer of July 2019 in the Roadstead of Brest, France. The water depth is around $10 \mathrm{~m}$ and up to 5 transmission ranges between $[65,540] \mathrm{m}$. Each channel transmission was performed by using a 511-Maximal Length Sequence (MLS) as probe signal [16] centered on $f_{c}=27 \mathrm{kHz}$ over a $6 \mathrm{kHz}$ bandwidth. Fig. 1 provides an example of the delay-Doppler spread extracted from the successive estimated Channel Impulse Response (CIR). Estimated channel delay spread and Doppler spread are reported in Table I.

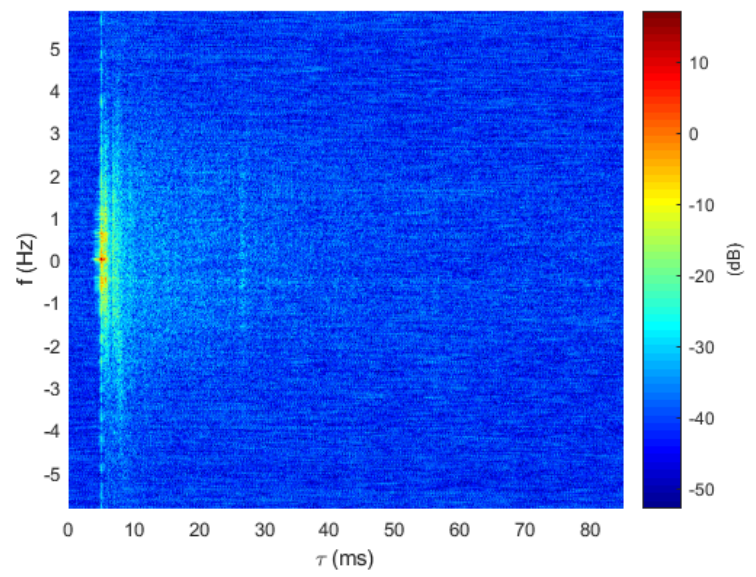

Fig. 1: Delay-doppler spread function for the roadstead of Brest with $D_{1}=200 \mathrm{~m}$.

\section{B. Watermark replay channel}

To simulate a real experiment, we consider in this section the Watermark channel [12] which is a replay channel simulator driven by measurements of the time varying CIR. The principle of the simulator consists of distorting input waveforms by convolving them with measured channels. To simulate a multiuser communication, we sum the output of several Watermark channel fed by different CIRs and delayed by relative range of each user. For a mobile multiuser communication, the operation of channel replay in the Single Input Single Output (SISO) case can be expressed in baseband as:

$$
\begin{gathered}
r(t)=\sum_{\substack{i=1 \\
e_{u}}}^{N^{2 \pi f_{c} a_{i}(t-\tau)} d \tau+n(t)} \hat{h}_{-\infty}^{+\infty}(\tau, t) s_{i}\left(\left(1-a_{i}\right)\left(t-\tau-\bar{\tau}_{i}\right)\right) \ldots \\
\end{gathered}
$$

where the Doppler shift is artificially added in the receive signal by resampling and phase rotating the transmitted signal. In the following, the Doppler shift will be the same for all multipaths. $s_{i}(t)$ is the input signal, $\hat{h}_{i}(\tau, t)$ is the recorded CIR of the $i$-th user, $\bar{\tau}_{i}$ is communication delay between the $i$-th user and the receiver and $n(t)$ is a Gaussian noise.
Roadstead of Brest channel parameters are summarized in Table I whereas transmission system parameters are provided in Table II.

\begin{tabular}{|l|c|r|}
\hline Symbol & Signification & Value \\
\hline$f_{c}$ & Center frequency & $27 \mathrm{kHz}$ \\
\hline$f_{s}$ & Sampling frequency & $96 \mathrm{kHz}$ \\
\hline$B$ & Signal bandwidth & $4 \mathrm{kHz}$ \\
\hline$D_{i}$ & Transmission range & $10 \mathrm{~m}$ \\
\hline$z_{w}$ & Water depth & $10 \mathrm{~dB}$ \\
\hline SNR & Signal to noise ratio & $\mathrm{m}$ \\
\hline$\tau_{\max }$ & RMS channel delay spread $[17]$ & {$[8.85,26.49] \mathrm{ms}$} \\
\hline$\sigma_{\max }$ & RMS channel Doppler spread [17] & {$[0.85,2.9] \mathrm{Hz}$} \\
\hline
\end{tabular}

TABLE I: Watermark channel parameters.

\begin{tabular}{|l|c|r|}
\hline Symbol & Signification & Value \\
\hline$M$ & Modulation order & 2 (DBPSK) \\
\hline$N_{s}$ & Number of symbols per frame & 200 \\
\hline$N_{f}$ & Number of frames & Convolutive code \\
\hline $\mathcal{C}$ & FEC code type & $(133,171)_{o}$ \\
\hline$g_{\mathcal{C}}$ & FEC code generator & $\frac{1}{2}$ \\
\hline$R_{\mathcal{C}}$ & FEC code rate & $31.3 \mathrm{~ms}$ \\
\hline$T_{g}$ & Guard interval time & 0.02 \\
\hline$\zeta$ & Orthogonality constant & $31.75 \mathrm{~ms}$ \\
\hline$T_{h}$ & Duration of the chirp signal & $0.25 \mathrm{~ms}$ \\
\hline$T_{c}$ & Chip duration & 0.25 \\
\hline$f_{l}, f_{h}$ & Bounds of HFM signal & $6 \mathrm{kHz}, 10 \mathrm{kHz}$ \\
\hline$\alpha$ & Pulse shaping filter roll-off factor & 127 \\
\hline$T_{s}$ & Symbol duration & $31.75 \mathrm{~ms}$ \\
\hline$N_{S F}$ & Spreading factor &
\end{tabular}

TABLE II: System parameters.

\section{Performance metrics}

To compare the performance of the proposed method, we will use as benchmark TDMA with Direct Sequence Spread Spectrum (DSSS), CDMA and MU-CSS protocols [8]. Spreading factors of TDMA DSSS and CDMA are chosen equal to time-bandwidth product of MU-CSS and the proposed MU-HFM waveforms such as performance of all techniques are comparable to the single user scenario. The TDMA guard interval time is chosen to be greater than the maximum duration of the various channel delays.

The chosen performance metric is the average effective data rate per user defined for each protocols family as follows [17]:

$$
\begin{gathered}
D_{e}^{\mathrm{CDMA}}=\frac{R_{\mathcal{C}} \log _{2} M}{N_{S F} \cdot T_{c}} \cdot(1-\mathrm{FER}) \quad[\mathrm{bps}] \\
D_{e}^{\mathrm{TDMA}}=\frac{R_{\mathcal{C}} \log _{2} M}{N_{u} N_{S F} T_{c}+\left(N_{u}-1\right) T_{g}} \cdot(1-\mathrm{FER}) \quad[\mathrm{bps}] \\
D_{e}^{\mathrm{MU}-\mathrm{CSS}}=D_{e}^{\mathrm{MU}-\mathrm{HFM}}=\frac{R_{\mathcal{C}} \log _{2} M}{T_{h}} \cdot(1-\mathrm{FER}) \quad[\mathrm{bps}]
\end{gathered}
$$

where $M$ is the size of the DPSK constellation, $R_{\mathcal{C}}$ is channel coding rate and FER is the Frame Error Rate. A frame is considered erroneous when at least one bit per frame after channel decoding is erroneous. 


\section{Performance results with mobile users}

AUVs motion is emulated by adding motion-induced Doppler scale at the output of Watermark channel. For each frame, speed value of each AUV is randomly selected in the interval $[-2,2] \mathrm{m} / \mathrm{s}$.

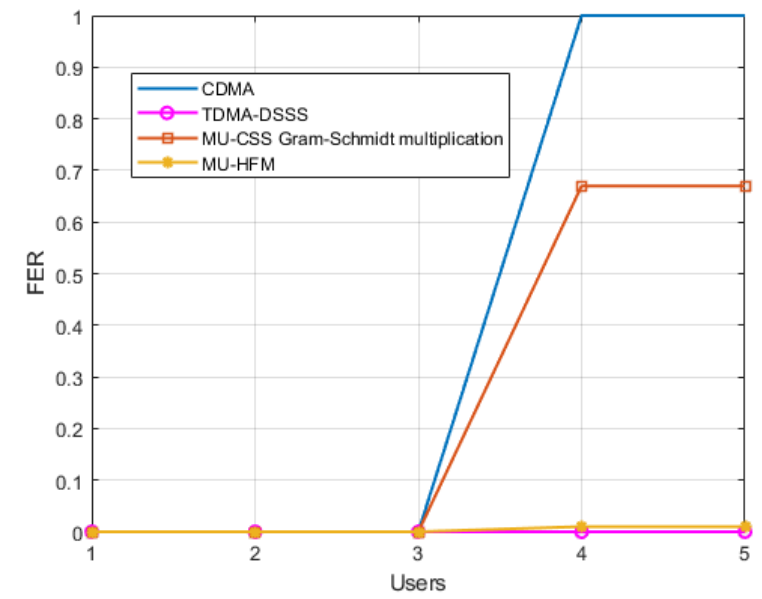

Fig. 2: Average FER performance versus number of users for the replayed channel of the roadstead of Brest with Doppler shift known.

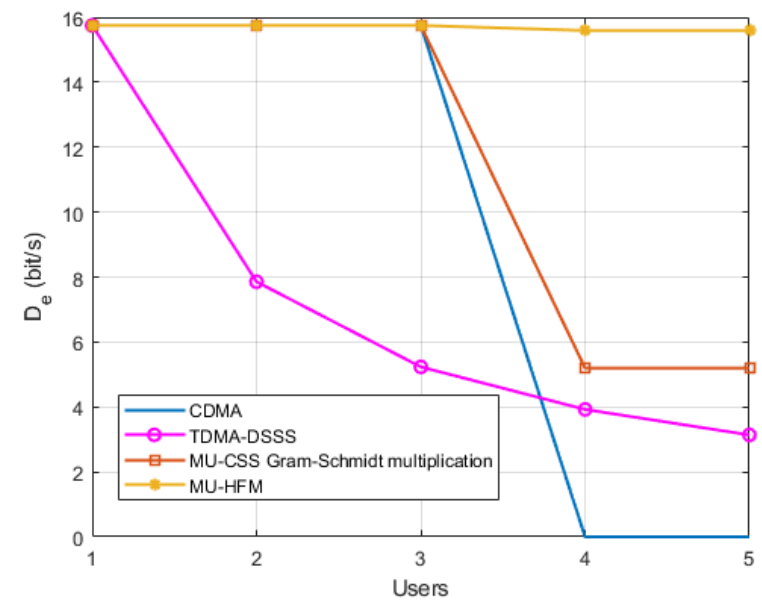

Fig. 3: Average effective data rate per user versus number of users for the replayed channel of the roadstead of Brest with Doppler shift known.

Fig. 2 and Fig. 3 show the performance of the different protocols with perfectly compensated Doppler shift. In the single-user scenario, the different protocols provides a Frame Error Rate (FER) of $0 \%$ and therefore a maximum data rate. Up to 3 users, CDMA, MU-CSS multiplication [11] and MU-HFM have the same FER. This means that they also have the same data rates. However, we can see TDMA has a decreasing data rate despite of a FER of $0 \%$ because of waiting times between communications. From 4 users, CDMA provides an FER of $100 \%$ due to multiple access interference terms and consequently a data rate of $0 \%$. The MU-CSS provides lower FER due to its orthogonal construction but is outperformed by the proposed MU-HFM technique. Thanks to a near-zero FER, the data rate of MU-HFM is almost equal to the maximum data rate which is obtained for 1 user. This phenomenon can by explained by the fact the Hadamard codes of MU-CSS have been replaced in MU-HFM by orthogonal signals similar to HFMs which are more resistant to UWA channel effects such as Doppler spread and multipath effect.

\section{CONClusion}

In this article, we have proposed a new multiuser technique based on a set of chirp related to orthogonal HFM signals denoted MU-HFM. The UWA communication was carried out in the most realistic case with a fleet of AUVs in motion. Experimental results with Watermark channel fed by sea channel sounding show the superiority of MU-HFM in a realistic scenario and constitutes a good solution for multiuser communications with mobile users navigating in different directions.

In the future, we study the performance of the proposed technique for higher number of users and explore the impact of non perfect Doppler shift estimation to the decoder.

\section{ACKNOWLEDGMENT}

The authors thank Brittany Region (France) and Thales DMS France for their support.

\section{REFERENCES}

[1] B. T. Champion and M. A. Joordens, "Underwater swarm robotics review," 2015 10th System of Systems Engineering Conference (SoSE), pp. 111-116, May 2015.

[2] M. Stojanovic and P.-P. J. Beaujean, "Acoustic Communication," in Springer Handbook of Ocean Engineering, M. R. Dhanak and N. I. Xiros, Eds. Springer International Publishing, 2016, pp. 359-386.

[3] F. Qu, Z. Wang, L. Yang, and Z. Wu, "A journey toward modeling and resolving doppler in underwater acoustic communications," IEEE Communications Magazine, vol. 54, no. 2, pp. 49-55, February 2016.

[4] R. Otnes, A. Asterjadhi, P. Casari, M. Goetz, T. Husøy, I. Nissen, K. Rimstad, P. v. Walree, and M. Zorzi, Underwater Acoustic Networking Techniques, ser. SpringerBriefs in Electrical and Computer Engineering. Berlin Heidelberg: Springer-Verlag, 2012. [Online]. Available: //www.springer.com/la/book/9783642252235

[5] J. Yin, G. Yang, D. Huang, L. Jin, and Q. Guo, "Blind adaptive multi-user detection for under-ice acoustic communications with mobile interfering users," The Journal of the Acoustical Society of America, vol. 141, pp. EL70-EL75, 012017.

[6] T. C. Yang, "Spatially Multiplexed CDMA Multiuser Underwater Acoustic Communications," IEEE Journal of Oceanic Engineering, vol. 41, no. 1, pp. 217-231, Jan. 2016.

[7] F. Yuan, Q. Wei, and E. Cheng, "Multiuser chirp modulation for underwater acoustic channel based on VTRM," International Journal of Naval Architecture and Ocean Engineering, vol. 9, 102016.

[8] C. Bernard, P.-J. Bouvet, A. Pottier, and P. Forjonel, "Multiuser chirp spread spectrum transmission in an underwater acoustic channel applied to an AUV fleet," Sensors, vol. 20, 032020.

[9] S.Lang, Introduction to Linear Algebra. Springer-Verlag New York Inc., December 1985

[10] E. Kaminsky, "Chirp signaling offers modulation scheme for underwater communications," SPIE Newsroom, 2006. [Online]. Available: http://www.spie.org/x8626.xml

[11] C. Bernard and P.-J. Bouvet, "Multiuser underwater acoustic communication for an AUV fleet," in OCEANS 2019 MTS/IEEE, Marseille, France, Jun. 2019. 
[12] P. Van Walree, F.-X. Socheleau, R. Otnes, and T. Jenserud, "The Watermark Benchmark for Underwater Acoustic Modulation Schemes," IEEE Journal of Oceanic Engineering, vol. 42, no. 4, pp. 1007 1018, Oct. 2017. [Online]. Available: https://hal.archives-ouvertes.fr/hal01630274

[13] Y. M. Aval and M. Stojanovic, "Differentially coherent multichannel detection of acoustic OFDM signals," IEEE Journal of Oceanic Engineering, vol. 40, no. 2, pp. 251-268, April 2015.

[14] M. Stojanovic, "Underwater Acoustic Communications: Design Considerations on the Physical Layer," in 2008 Fifth Annual Conference on Wireless on Demand Network Systems and Services, Jan. 2008, pp. $1-10$.

[15] A.Ralston, A First Course in Numerical Analysis. Dover Publications Inc, February 2001.

[16] P. van Walree, "Channel sounding for acoustic communications: Techniques and shallow-water examples," Norwegian Defence Research Establishment (FFI), Tech. Rep. FFI-rapport, vol. 7, 2011.

[17] J. G. Proakis and M. Salehi, Digital Communications 5ed, 5th ed. Boston, Mass.: McGraw-Hill, 2008. 